\title{
VII. Friedrich Fedde, Neue Arten von Corydalis aus dem Himalaya und den angrenzenden Teilen von Tibet. I.
}

1. Corydalis urosepala fedde spee. nov. - Herba, ut videtur, perennis atque elata radice..., caule erecto, ramoso, multangulo. - Folia basalia..., caulina petiolata, subvaginata, bipinnatisecta, segmentis petiolulatis, rarius modo trilobatis, plerumque trisectis, lobis rursus bi- vel trilobulatis, ad basim eleganter cuneatis, lobulis oblongo-obovatis, saepius mucronulatis. - Flores videntur lutei, in racemos terminales et laterales, in partibus junioribus densifloros, nisi capituliformiter aggregatus, postea laxiores, multifloros, laterales foliis breviores, terminalem folia superantem, dispositi; apex capituliformis racemi videtur villosus caudiculis scpalorum longis alabastra multo superantibus; bracteae lineari-lanceolatae, angustae, longe acuminatae, subintegrae, denticulis sparsis atque inter se remotis armatae, $0,5-0,75 \mathrm{~cm}$ longae, pedicellos primo perbreves, deinde longiores superantes. - Sepala in genere eximia, 1,0-1,2 cm longa, infra ovato-subcordata, trinervia, fimbriolata, satis subito in apicem caudiciformem apiculata, ceterum autem pellucida, decidua; petalum superius $1,75-2 \mathrm{~cm}$ longum, angustum, in tato vix subsigmoideum, ad apicem subito satis longe apiculatum, marginibus non ita evalutis, sed fimbriatula-crenulatis ambitu anguste oblongum, ad dorsum modo in parte anteriore carinae crista perhumili et brevi, denticulata ornatum, calcari minus quam dimiduum $(\% / \%)$ totius petali occupante, subcylindrico, ad apicem paulo subbullatum leviter decurvato; petalum inferius lamina lccurvata simile, ungue subbullato et ad basim gibberulo minima ornato; petala interiora pro genere satis angusta, anguste oblonga, ad basim oblique subtruncato-angustata, ad dorsum crista intermedia paullo infra apicem incipiente, humili, paulatim usque ad unguis lincari-lancolati bisim decrescente ornata; staminum adelphiae filamenta lincari-lanceolata, supra in summum tertium filiformiter angustata, appendix nectarifer us longus, ad apicem leviter declinatum leviter incrassatus, fere totum calcar percurrens; ovarium anguste lanceolato-oblongoideum, paulatim in stylum aequilongum angustatum, stigmate cupitato-bilobo, sexstipitato. - Siliqua (immatura) declinata, anguste lineari-oblonga, ut videtur torulosa.

Zentralasiatisches Gebiet: Provinz des Extratropischen Himalaya; westlicher Himalaya. - Chitral (S. M. Toppin no. 359 !).

Nota: Inter omnes praeclara sepalis pro genere permagnis, tam longe caudatis, ut summo in racemo caudiculac sepalorum alabastrorum capituliformiter congestorum fasciculum. penicillatum forment; ceterum elucet petalis exterioribus distincte apiculatis marginibus fimbriatulo-crenulatis, crista intermedia petalor um interiorum demum paullo infra apicem incipiente at usque ad unguis basim perveniente. Affinitas dubia mihi videtur: fortasse maxime ad C. Hookeri convenit. 
2. Corydalis strachoyioides Fedde spec. nov. - Herba perennis, minus elata, 10-20 cm alta, glaucescens, rhizomate infra diviso, robusto, elongato, caulibus foliosis, flexuosis, infra decumbentibus, ramosis. Folia basalia, ut videtur, mox marcescentia, cetera longipetiolata, petiolis leviter vaginantibus, bipinnatisecta, segmentis petiolulatis pinnatipartit's, lobis 3-5-fidis, lobulis oblongis vel obovato-oblongis, breviter acutis vel subrotundatis, plerumque submucronulatis, subtus papilloso-villosulis. - Flores lutei (?) in racemos terminales et axillares. multifloros breves dense congesti; bracteae infimae pinnatipartitae vel trifidae, superiores integrae anguste lineares; pedicelli breviores, past anthesim elongati et deflexi. - Sepala diminuta, squamoidea, rotundato-cordato, denticulata, decidua; petalum superius $1,2-1,3 \mathrm{~cm}$ longum, subrectum, ad apicem rotundatum, marginibus mediocriter evo'utis ambitu oblongo-orbiculare, ad dorsum crista ab apice paulatim adscendente usque ad calcaris finem paulatim decrescente ornatum, calcari dimiduum totius petali occupante, subcylindrico, subrecto, ad apicem rotundato-obtusum paullo declinatum; petalum inferius lamina ambitu rotundato-oblonga carinatum, ad apicem subemarginatum, crista tum humillima vel subnulla, tum satis alta, rcpanda vel repando-denticulata, ungue iterum subcarinato, ad basim gibberulo parvo ornato; petala interiora lamina oblongo-subpanduraeformi, ad basim truncata, ad dorsum crista supra sacculatim dilatata, satis alta, in unguis initio evanescente, saccula laterali triangulari, ungue anguste obcuneato; staminum adelphiae filamenta in parte inferiore lanceolato-carinata, appendix rectus, ad apicem vix incrassatus, dimiduum calcaris percurrens; ovarium anguste oblongoideum, satis subito in stylum fere duplo longiorem, satis robustum angustatum, stigmate bilobo, octostipitato. - Siliqua oblongoidea, stylo abrupte terminata.

Innerasiatisches Gebiet; Provinz des extratropischen Himalaya; Östlicher Himalaya. - Bhutan (westlichster Teil): Chugya, 15000' (Rohmoo Lepcha 1912 no. 279!).

Nota: Valde affinis $C$. Stracheyi, a qua differt foliorum lobulis latioribus, racemis densioribus ac brevioribus, apice petalorum exteriorum rotundato vel subemarginato, ovario minus lato, stylo longiore et stigmate octo stipitato. Etia $m$ in hac specie variant cristae imprimis petali inferioris, quae sunt interdum subnullae.

3. Corydalis adiantifolia Hk. f. et Th. var. dentipetala Fedde var. nov. - Folia, ut videtur, etiam magis quasi puberula. - Conglomeratio bractearum ad racemorum apjces crinitarum imprimis densa atque distincta. - Petalor um exteriorum margines et cristae serrato-denticulatae. - Kaschmir: Baltistan, Tsumgáks to Pón (Schlagintweit fl. fr. VII. 1856 no. 6057 !).

Nota: Schlagintweit no. 5574 ex Skárdo format denticulatione petalorum minus evoluta transitum formae typicae ad varietatem.

4. Corydalis nana Prain var. Jaquemontii Fedde var. nov. - C. aurea Jacquemont in herb., non Willd. - Differt racemis laxifloris, magis elatis, bracteis minus divisis, summis integris; cristis petalorum exteriorum 
altioribus. - Westlicher Himalaya: Hangarang. (?) (Jacquemont no. 729 !) ad rivulos, alt. $3800 \mathrm{~m}$. - Garhwal: (Falconer no. 127 pro parte!). Damdar Valley, 11-12000 m (Duthie, fl. VII. 1883 no. 949!), Dudu Gádh (Duthie no. 949a!).

Nota: Altitudo et longitudo cristae valde varians. Fortasse species propria!

5. Corydalis Gerdae Fedde ${ }^{1}$ ) spec. nov. - Herba perennis, subcaespitosa, florum numero et magnitudine amoena, 10-20 om alta, breviter papilloso-villosula, rhizomate longo, atrobrunneo, subrepente, reliquiis petiolorum marcidorum obtecto, caulibus flaccidis teneris, infra nudis, modo in tertio summo duobus foliis oppositis instructis, racemis subumbellatis terminatis. - Folia basalia et caulina duo conformia longissime petiolata, petiolis ad basim vaginanter dilatatis, biternati-usque ad bipinnatisecta, segmentis petiolulatis, lobis in 3-5 lobulos obovato-oblongos acutos vel subrotundatis profunde fissis, imprimis subtus papilloso-villosula. - Flores speciosi suberecti, colore ignoto, ad apicem autem obscurius colorati, 4-7 in racemos terminales subumbellatim densissime congestos, folia satis superantes; bracteae e basi cuneata flabellatim quinquefidae, lobo terminali saepe iterum trifido, pedicellos breves superantes. Sepala minima, vix $0,5 \mathrm{~mm}$ longa, $1 \mathrm{~mm}$ lata, semicirculari-amplexicaulia, denticulata, squamaeformia, pellucida; petalum superius subrectum $1,75 \mathrm{~cm}$ longum, ad apicem acutum submucronulatum marginibus auriculatim expansis, infra constrictis, ad dorsum crista ardue adscendente supra carinam alta, postea angustissima usque ad calcaris finem perreniente ornatum, calcari $1 / 4-1 / 5$ totius petali occupante, subcylindrico, leviter decurvo, obtuso; petalum inferius lamina marginibus satis expansis et declinatis ambitu suborbiculari, orista alta semilunari carinae affixa, ungue carinato-bullato triplo longiore et etiam duplo latiore; petala interiora subpanduraeformi-oblonga, ad basim truncata, ad dorsum crista intermedia apice saccato-subinflato paullo super petali apicem prominente supra mediam. laminam altissima, in medio ungue lineari lamina duplo longiore paulatim evanescente, duobus sacculis lateralibus, superiore triangulari, inferiore plano; staminum adelphiae filamenta in summo quarto anguste linearia infra late carinato-naviculaeformia; ovarium oblongoideum, jam immaturum, ut videtur. subtorulosum, paulatim in stylum multo breviorem angustatum, stigmate bilobo infra bicornuto. Siliquam non vidi.

Zentralasiatisches Gebiet; Provinz des extratropischen Himalaya; östlicher Himalaya. - Bhutan (an der Grenze von Tibet und Phari): Ohumolari; 16000' (Roh moo Lepcha, Fl. East Himal. fl. IX. 1912 no. 525 !).

Nota: Species valde amoena floribus speciosis dense congestis, verosimiliter adspectu Ericae specierum grandiflorarum et decus declivitatum

1) In honorem filiae meae Gerda fieddr, scientiae amabilis studis minus deditae, quam flores amoenos ac splendidos amantis. 
alpinarum. Notabilis floribus in tato quasi ventricosis umbellatim dispositis, crista petali superioris usque ad calcaris finem perveriente, crista intermedia petalorum interiorum medio in ungue demam desinente, filamentis naviculaeformibus, bracteis flabellatis, quibus congruit cum (:. longipede affini.

6. Corydalis Onobrychis Fedde in Fedde, Rep. X (1912) p. 565. Adde: Bracteae infimae foliorum segmentis similes, superiores trifidae usque ad lineari-lanceolatae. - Weitere Fundorte: NW.-Kaschmir, Burji la $13000^{\prime}$ (A. Meebold, Fl. NW.-Himal. fl. VIII. 1905 no. 2999 pro parte!). Chitral, Madaglast $11000^{\prime}$ (S. M. Toppin no. 805!).

7. Corydalis onobrychoides Fedde spec. nov. - Herba perennis, ut videtur, minus robusta, glauca, e radice perpendiculari elongata enascens, ad collum reliquiis vaginarum obtecta, caulibus compluribus adscendentibus subnudis, striato-angulatis, circiter $20 \mathrm{~cm}$ altis. - Folia petiolata, omnia fere basalia, longe petiolata, petiolis basim versus vaginatis, subhipinnatisecta, segmentis distantibus, alternis vel oppositis, longius vel hrevius petiolulatis, flabellatim vel palmatim pinnatipartitis, lobulis obovato-oblongis, subacutis, marginibus non inter se tegentibus, vix cmergentiis parcissimis villosula, petiolis inclusis $7,5-15 \mathrm{~cm}$ longis, caulina pauca magis reducta, brevius petiolata. - Flores primo adscendentes, deinde descendentes, in racemum terminalem primum densissimum, subcapituliformem, deinde paullo laxiorem folia superantes compressi; bracteac omnes integerrimae, anguste obovato- vel rhomboideo-lanceolatae usque ad ablongo-lanceolatae, pedicellos valde superantes et alabastra obtegentes, 1-2 cm longae. - Sepala membranacea, squamoidea, valde diminuta, a mbitu irregularia, late sessilia; petalum superius circiter $2 \mathrm{~cm}$ longum, subrectum, ad apicem subadscendentem acutum, crista semilunari a summo apice oriente supra carinam ornata, calcari fere dimiduum petali occupante, paulatim decurvato, subcylindrico, rotundato-acuto; petalum inferius ad apicem profunde carinatum et paullo altius quam petalum supcrius cristatum, marginibus vix expansis, ungue distincte contractione separato, rursus carinato et ad basim minime gibberulo; petala interiora ungue obovato-lanceolato, quam lamina breviore, lamina subpanduraeformi-obovata, ad apicem late rotundata, ad basim emarginata, ad dorsum crista sacculaeformi in unguis initio finiente ornata; staminum adelphiae filamenta infra late lanceolata, in medio nervo bullatim emergente, appendix rectus, ad apicem incrassatus, dimiduum calcaris percurrens; ovarium oblongoideum, stylo aequilongum, stigmate bilobum, ad apicem bistipitatum, ad basim subbicornutum. - Siliquam non vidi.

Zentralasiatisches Gebiet; Provinz des extratropischen Himalaya. - Chitral; (Toppin no. 759!). - NW.-Kaschmir: Baltistan, Burji la $13000^{\prime}$ (A. Meebold, Fl. NW.-Himal. fl. VIIr. 1905 no. 2999 pro parte!).

Nota: A me primum cum $C$. Onobrychide valde affini confusa (in exemplaribus Meeboldianis commixtis), sed differt primo adspectu lobulis foliorum multo latioribus et maioribus, non marginibus inter se tegentibus, 
et bracteis magnis integerrimis, alabastra tegentibus; in tato etiam videtur minus rabusta, calcari graciliore, stigmate bistipitato, gibberulo ad basim petali inferioris.

8. Corydalis Schlagintweitii Fedde in Fedde, Rep. XIII (1914) 303. - Adde de affinitate: Et habitu et effiguratione floris; imprimis calcari brevissimo et crista intermedia petalorum interior um, denique bracteis lanceolatis $C$. strictae valde affinis, a qua differt floribus densissime confertis, cristis petalorum exteriorum humilioribus brevioribusque integris, modo ad summnm apicem petalorum effiguratis.

\section{Friedrich Fedde, Neue Arten aus der Verwandtschaft der Corydalis aurea Willd. von Nordamerika. $\left.X^{\mathbf{1}}\right)$.}

35. Corydalis euchlamydea (Wooton et Standley) Fedde nom. nov. Syn.: Oapnoides euchlamydeum Wooton et Standley in Contrib. U. S. Nat. Herb. XVI. 4 (1913). - Die Autoren soheinen merkwürdigerweise meine neuen Veröffentlichungen über die nordamerikanischen Arten nicht gekannt zu haben, denn sie hätten sonst wohl die oben erwähnte Art mit den von mir beschriebenen verglichen, bzw. die Verwandtschaft festgestellt.

Nach Angabe der Autoren ist sie, wie dies ja auch ziemlich selbstverständlich ist, mit $C$. aurea verwandt, untersoheidet sioh von ihr aber durch sehr große Tragblätter und durch nahe an den Grund der Blattstiele gerückte Fiederpaare. Auch handelt es sich um eine ziemlich zarte Pflanze mit mehr geschlitzten Blättern.

Neu-Mexika: An mehreren Stellen (Sacramento Mts. 1890, James Cañon 1899, Ruidoso Creek 1895) von Woot on gesammelt. Dgl. Clonder oft (Stearns no. 348).

Am näohsten verwandt erscheint mir meine $C$. curvisiliquaeformis (Rep. XI [1912] 289). Diese hat a ber keine zarten, sondern kräftige Sténgel, auch habe ich Tragblätter gesehen, die höchstens $10 \mathrm{~mm}$, nicht aber bis $25 \mathrm{~mm}$ lang waren. Sehr gut stimmen aber die Beschreibungen des Blattgrundes, der Blüte und der Frucht gowie der Samen überein, so da B man nur die Ungenauigkeit bei der Beschreibung der einzelnen Blütenteile bedavern kann, obne deren genaue Bchilder ung sich nun mal die einzelnen Arten der Gattung nicht auseinander halten lassen.

36. Corydalis aurea Willd. - Die Standortsangaben von Wooton und Standley l. c. XIX (1915) 263 ersobeinen mir insofern zweifelhaft, als es sich wohl nicht um $C$. aurèa selbst, sondern um Verwandte $(C$. montana, $C$. Jonesii, C. tortisiliqua) handeln dürfte.

1) Cf. Rep. X, 311, 364, 379, 417, 479; XI, 196, 289, 497; XII, 37. 